\title{
Effect of ultrasonic treatment on the properties of multiwalled carbon nanotubes - polymethylmethacrylate composites: Effect of applied voltage and pressure on conductivity of the composites
}

\author{
S. I. Moseenkov ${ }^{1 *}$, V. L. Kuznetsov ${ }^{1,2}$, G. V. Golubtsov ${ }^{1,2}$, A. V. Zavorin ${ }^{1,2}$, A. N. Serkova ${ }^{1}$ \\ ${ }^{1}$ Federal Research Center Boreskov Institute of Catalysis, Lavrentiev Ave. 5, 630090 Novosibirsk, Russia \\ ${ }^{2}$ Novosibirsk State University, Pirogova str. 1, 630090 Novosibirsk, Russia
}

Received 12 April 2019; accepted in revised form 27 June 2019

\begin{abstract}
The effect of sonication time during the synthesis of multiwalled carbon nanotubes - polymethylmethacrylate (MWCNT/PMMA) composites by coagulation technique on the MWCNT distribution in the bulk of the composites close to the percolation threshold was systematically studied by SEM and by measurements on specific resistivity in a wide range of voltages and external pressure. It was found that the resistivity of composites is irreversibly reduced up to $10^{5}$ times during the first 1-3 measurements, while further measurements are characterized by high repeatability. Change of the composite resistivity during the measurements is discussed assuming that several types of contacts between the nanotubes changing during the measurement are present. It was found that an increase of the sonication time changed the ratio between ohmic and non-ohmic contacts. It affected the type of changes in the sample resistivity during the voltage variation in the range of $0-10^{3} \mathrm{~V} / \mathrm{mm}$ and resulted in a nonuniform dependence of the composite specific resistivity on the sonication time. An increase of the composite volume during the resistivity measurements was observed at the current densities above $4 \cdot 10^{-8} \mathrm{~A} / \mathrm{cm}^{2}$. New ohmic contacts were found to form when external pressure was applied due to squeezing of the polymer matrix from the space between adjacent nanotubes.
\end{abstract}

Keywords: nanocomposites, multiwalled carbon nanotube, polymethylmethacrylate, contacts between nanotubes, currentvoltage characteristic

\section{Introduction}

Due to their unique electrophysical properties, carbon nanotubes (CNT) are promising materials for development of composite and functional materials with improved operational properties [1-4]. The CNT introduction in polymer and ceramic matrices can lead to significant increase of their electrical conductivity, which opens new prospects for practical application of the synthesized composites, e.g. as sensitive elements of cost-efficient deformation probes [5-7], as light materials with antistatic properties, for improvement of electromagnetic compatibility in various devices [8-11] and in many other applications.

The conductivity of composites is known to depend on the CNT concentration in the composite and method of the composite preparation [12,13]. Using the theory of percolation, it was shown that with a uniform distribution of long nanotubes that are not agglomerated and that do not interact with the composite matrix, the percolation threshold is reached already at the CNT concentration 0.01-0.1 vol\% [14]. A significant deviation of the percolation threshold either to larger or to smaller values indicates

*Corresponding author, e-mail: moseenkov@gmail.com C BME-PT 
CNT structuring in the composites (existence of preferable nanotube orientation, presence of aggregates, surface filling of the matrix structural elements as in ceramic matrices, etc.) or the interaction of the composite matrix with the CNT surface resulting in partial blocking of contacts between individual nanotubes (as in polymer matrices) [15-17].

Coagulation technique is one of the methods used for production of composites with MWCNTs. Due to the fact that in the process of obtaining composites by the method of coagulation technique, the uniformly mixed polymer and filler are deposited from the stable suspension 'filler-polymer-solvent' by adding an auxiliary solvent, reducing the stability of the suspension, the initial state of the filler can be retained. It makes possible to regulate the filler distribution in the polymer matrix [4].

The effect of the ultrasonic treatment parameters on deagglomeration and dispersion of nanotubes was studied in detail [18-20]. It was found that it was possible to provide different ratios between aggregates and dispersed MWCNTs and vary the aspect ratio of the nanotubes by varying the power and duration of the treatment. The role of the surface functional composition of MWCNT and the used polymer matrix is also important. It was shown that the presence of functional groups on the MWCNT surface blocks the formation of contacts between the nanotubes [21]. Their elimination from the MWCNT surface promotes the formation of contacts between the nanotubes and increases their conductivity by as much as 4 orders of magnitude. An increase of the adhesion energy between the polymer and the CNT surface also stimulates blocking of contacts between the nanotubes due to the formation of films from polymer molecules at places of potential contacts [22].

Variation of the MWCNT concentration, functional composition of their surface, ultrasound treatment parameters and the used polymer matrix may lead to changes of both the absolute number of contacts between the nanotubes and ratio of different types of contacts ultimately determining the composite properties.

Investigation of the current-voltage characteristics (CVC) of MWCNT composites is one of the methods for evaluation of the composite structure and properties. A deviation of the conductivity from the ohmic dependence was observed for composites near the percolation threshold. It was attributed both to the presence of the Coulomb gap between nanotubes in the composite [23-25] and to existence of the contact resistivity during the CVC measurement by a two-contact method [26]. In [27] the observed effect was attributed to resonance tunneling typical for layered semiconductors.

The purpose of this study was to obtain MWCNT/ PMMA composites with various types of nanotube agglomerate distribution in the polymer matrix (with a concentration of nanotubes close to the percolation threshold). To obtain composites using the co-coagulation method, we varied the ultrasonic treatment time during the preparation of nanotube powder suspensions and PMMA to affect the size of the nanotube agglomerates. SEM and optical microscopy were used to characterize the distribution of nanotubes in the PMMA matrix, and the CVC for characterizing the resistivity of the composites. Resistivity measurements in the voltage range of $0-10^{3} \mathrm{~V} / \mathrm{mm}$ show significant changes in the resistivity of the composite during measurements. The observed changes in resistivity are discussed under the assumption that there are several types of contacts between nanotubes that change during measurement. It was found that an increase in sonication time alters the relation between ohmic and non-ohmic contacts.

\section{Experimental}

\subsection{Synthesis of MWCNT-PMMA composites}

MWCNTs with the mean diameter of $9.8 \mathrm{~nm}$ were produced using the CVD method by ethylene decomposition with bimetallic $\mathrm{Fe}-\mathrm{Co} / \mathrm{A} 12 \mathrm{O} 3$ catalyst at $670^{\circ} \mathrm{C}[28,29]$. This catalyst with the active component $\mathrm{Fe}_{2} \mathrm{Co}$ has been shown to produce MWCNTs of low defectiveness [30] and with low content of inorganic impurities. Subsequent refluxing of nanotubes with $15 \% \mathrm{HCl}$ (followed by washing with distilled water until neutral $\mathrm{pH}$ and subsequent drying in air) allows decreasing the content of catalyst traces to $0.2-0.3 \mathrm{wt} \%$.

MWCNT/PMMA composites were prepared by the coagulation technique [31]. MWCNTs were added to PMMA (molecular weight 120000 , Sigma-Aldrich) solution in $\mathrm{N}, \mathrm{N}$-dimethylformamide and ultrasonicated (total suspension volume $150 \mathrm{ml}, 22 \mathrm{kHz}, 320 \mathrm{~W}$, USPA-0.4/22-OM, U-sonic, Russia) for different times in a temperature-controlled reactor $\left(20^{\circ} \mathrm{C}\right)$. To determine the percolation threshold of composites we varied the MWVNT content $(1,1.5,2,2.5,5$ and $8 \mathrm{wt} \%$ ), while the sonication time of each sample was 
fixed (30 min). To determine the effect of sonication time, composites with a fixed MWCNT content of $1.5 \mathrm{wt} \%$ and variable sonication time $\left(t_{\mathrm{s}}\right)$ of $3,5,7.5$, 10, 20, 30 and 45 minutes were prepared.

The produced suspensions were immediately introduced in an excess of water $\left(\sim 1.51,60^{\circ} \mathrm{C}\right)$. Then the coagulated composites were filtered, washed with distilled water to remove the solvent residues and successively dried in air and under vacuum $\left(10^{-1}\right.$ torr $=$ 1.33 mbar) at $90^{\circ} \mathrm{C}$. Thin films of PMMA composites were obtained using the hot press method at $180^{\circ} \mathrm{C}$ ( $5 \mathrm{~min}$ and $2 \mathrm{MPa}$ ). The thickness of the produced films was in range of $0.51-0.63 \mathrm{~mm}$. The samples were marked as C3, C5, C7.5, C10, C20, $\mathbf{C 3 0}$ and $\mathbf{C 4 5}$ according to ultrasonic treatment time.

\subsection{Investigation of distribution of MWCNTs by length}

To characterize the influence of ultrasonic treatment time on the length distribution of the MWCNTs, the suspension of nanotubes $(30 \mathrm{mg})$ in ethanol with $15 \mathrm{mg}$ of Triton X-100 (Sigma-Aldrich) was sonicated under the same conditions as the composites (total suspension volume $150 \mathrm{ml}, 22 \mathrm{kHz}, 320 \mathrm{~W}$, $20^{\circ} \mathrm{C}$ ). During the sonication of the suspension the samples of $100 \mu 1$ were taken at $3,5,10,20,30$ and 45 minutes. The samples were diluted with ethanol in a ratio of 1:70. The resulting suspensions with a low content of MWCNTs were sprayed on aluminum foil and quickly dried at $140^{\circ} \mathrm{C}$ to prevent secondary agglomeration of the nanotubes. The length distribution of MWCNTs for each sample was calculated on the base of 400-500 measurements of individual nanotubes in SEM images.

\subsection{Structural characterization}

The structure of MWCNT/polymer composites was characterized by optical microscopy (BioMed 5, Russia) and scanning electron microscopy (SEM, JSM6460LV Jeol, Japan). For characterization with optical microscopy, the samples were melted and spread over a slide of the optical microscope. For SEM characterization the composites were broken in liquid nitrogen, and the fracture surfaces were subsequently covered with a 5-10 nm gold layer.

\subsection{Resistivity and current-voltage characteristic measurements}

Picoamperemeter/voltage source Keithley 6487 (U.S.A.) with resistivity test fixture Keithley 8009
(U.S.A.) was used for measuring current-voltage characteristics in range of applied voltage $0-500 \mathrm{~V}$. The electrode application force in the test cell was $1-1.5 \mathrm{~N} / \mathrm{cm}^{2}$. For each voltage point the current measurement was performed after $60 \mathrm{~s}$ delay at fixed voltage. Average current was calculated from 50 measurements. If the measured current or applied voltage exceeded $1.5 \mathrm{~mA}\left(65 \mu \mathrm{A} / \mathrm{cm}^{2}\right)$ or $500 \mathrm{~V}$, respectively, the measurement was stopped and performed again for 3 cycles after $60 \mathrm{~s}$ delay at $0 \mathrm{~V}$ to remove a residual charge.

\subsection{Resistivity measurements under high pressure}

To measure the effect of the electrode pressure on the composite resistivity, a sample was placed between stainless steel electrodes $\left(5.31 \mathrm{~cm}^{2}\right)$ covered with a gold layer $(\sim 50 \mathrm{~nm})$. The electrodes were fixed in the clamps of a tensile-testing machine (Boreskov Institute of Catalysis, Russia) through insulating insets to eliminate their movement or shift. The sample was loaded with $420 \mathrm{~N} / \mathrm{min}$ rate, the voltage between the electrodes was set, and the current through the sample was measured using Keithley 6487. Measurements were performed without movement of the sample. The dependence of resistance on pressure was measured at the second and subsequent loading of the sample; the applied voltage was increased sequentially. The pause between the measurements was $3 \mathrm{~min}$. The installation described above was used to determine the effect of the current passing through the sample on the pressure of the sample on the measurement electrodes. Pressure about $100 \mathrm{~N} / \mathrm{cm}^{2}$ was applied to the sample, and relative location of the mobile and stationary parts of the tensile-testing machine was determined. The sample was kept in such state for $1 \mathrm{~h}$. The dependence of the electrode application force on time was registered directly before the measurement and for $20 \mathrm{~min}$ after the measurement for baseline correction. During the measurement the voltage between the electrodes was set using Keithley 6487. It was also used to measure the current passing through the sample. The average current was determined using moving mean filter by 300 points (at 1 reading per second rate). The force was registered with resolution of $0.01 \mathrm{~N}$. The holding time at fixed voltage was $300 \mathrm{~s}$. 


\section{Results and discussion}

\subsection{Effect of ultrasonic treatment time on the length of MWCNTs and size of their} aggregates in the composites

The change in the MWCNT length distribution as a function of the sonication time was examined using the SEM method. The obtained MWCNT mass distribution over the length and the change in average length of the MCNT as a function of ultrasonic treatment time are shown in Figure 1.

It was found that in the range of $t_{\mathrm{s}} 3-20 \mathrm{~min}$, the average MWCNT length is $1.6 \mu \mathrm{m}$, and the mass distribution of nanotubes by the length remains almost unchanged when using the ultrasonic processing mode

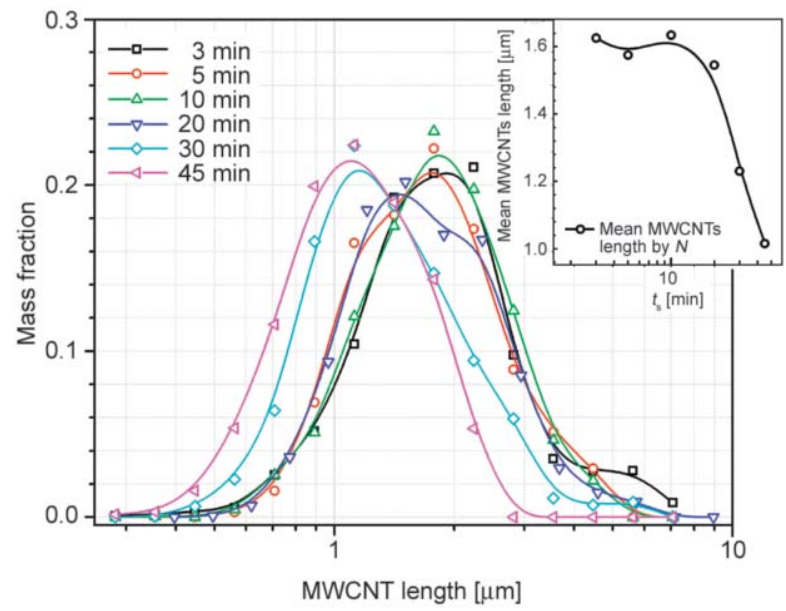

Figure 1. The dependence of the mass distribution of MWCNTs by length in suspension from the sonication time. Inset presents the effect of sonication time on the mean length of MWCNTs. $\left(150 \mathrm{ml}, 22 \mathrm{kHz}, 320 \mathrm{~W}, 20^{\circ} \mathrm{C}\right)$. The increase in $t_{\mathrm{s}}$ to $30-45 \mathrm{~min}$ leads to a decrease in the average MWCNT length to $1.0-1.2 \mu \mathrm{m}$ and practically to complete destruction of nanotubes more than 3$4 \mu \mathrm{m}$ in length.

The structure of the synthesized composites was studied by optical and scanning electron microscopy. Typical images obtained for composites with different ultrasonic treatment times are shown in Figure 2. Variation of the average size of observed MWCNT aggregates as a function of the ultrasonic treatment time was determined using optical microscopy. The perimeter of the aggregates was measured in optical images, and their average diameter was calculated. It was found that the average size of the aggregates steadily decreased during the ultrasonic treatment (from $60 \mu \mathrm{m}$ for $\mathbf{C 3}$ to $5 \mu \mathrm{m}$ for $\mathbf{C 4 5}$ ), whereas the coloration intensity and uniformity of the composite polymer matrix increased. Analysis of the SEM images of the composite fracture surfaces showed that molecules of the polymer matrix penetrated into the bulk of the initial MWCNT aggregates by no more than $0.5-1 \mu \mathrm{m}$. This observation made it possible to use the data on the quantitative size distribution of the aggregates for calculation of the aggregate weightsize distribution (Figure 3). The density of the aggregates was assumed to be equal to the MWCNT packing density $0.26 \mathrm{~g} / \mathrm{cm}^{3}$. The volume of the MWCNT aggregates was calculated assuming that they have spherical shape.

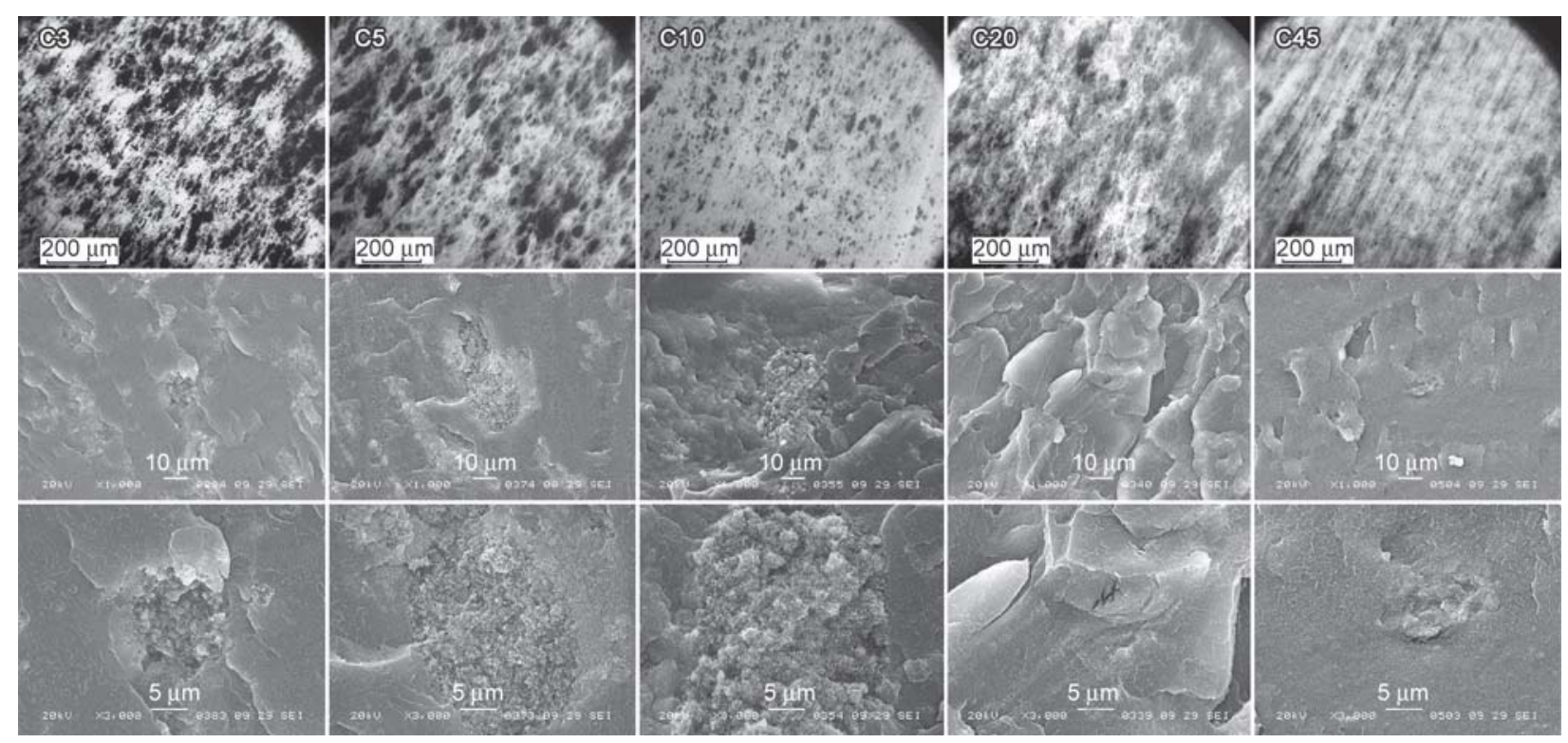

Figure 2. Typical images of the composite films (C3-C45 samples) obtained by optical microscopy (top row) and SEM images of composite fracture surfaces (middle and bottom rows; bottom row presents the same objects with higher magnification). 


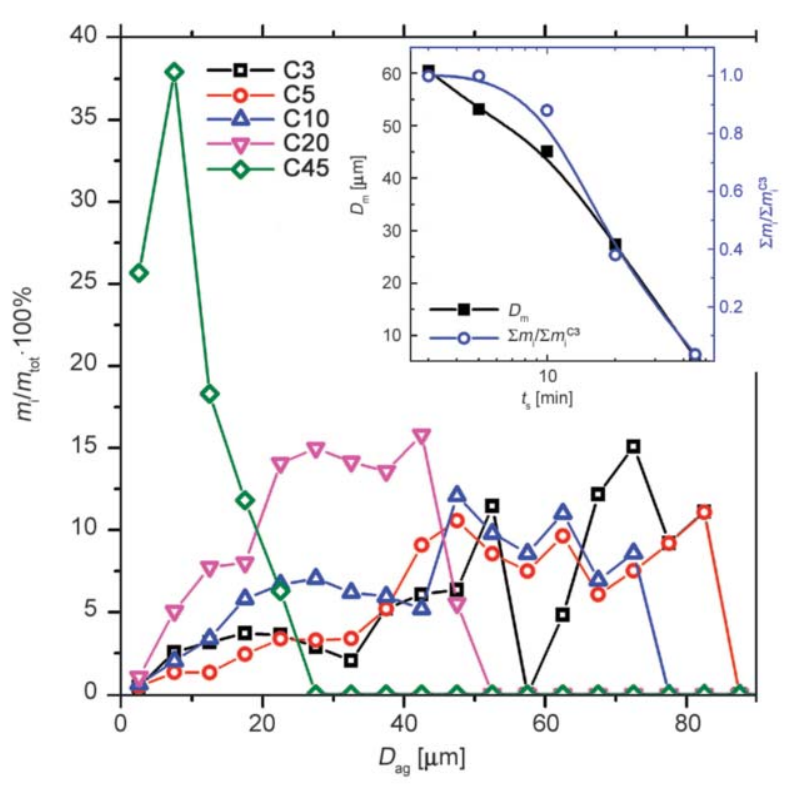

Figure 3. Changes of the weight-size distribution of MWCNT aggregates in the composites depending on the ultrasonic treatment time (according to the optical microscopy data). Inset - changes of the aggregate average size $\left(D_{\mathrm{m}}\right)$ and ratio of the mass of aggregates to the mass of aggregates observed in sample $\mathbf{C} 3$ versus the sonication time.

A wide weight-size distribution of agglomerates was observed for ultrasonic treatment with $t_{\mathrm{s}}=3-10 \mathrm{~min}$. When $t_{\mathrm{s}}$ was increased to $10-45 \mathrm{~min}$, the size distribution became narrower indicating that large aggregates were destroyed. The mass of observed aggregates calculated from the weight-size distribution and normalized relative to sample $\mathbf{C} \mathbf{3}$ (Figure 3, Insert) made it possible to estimate the degree of MWCNT dispersion during the ultrasonic treatment. At $t_{\mathrm{s}}=3-5 \mathrm{~min}$ the total mass of aggregates did not change much. When $t_{\mathrm{s}}$ was increased to $10-45 \mathrm{~min}$, this ratio $\sum m_{\mathrm{i}} / \sum m_{\mathrm{i}}^{\mathbf{C} 3}$ sharply decreased suggesting that the aggregates were destroyed, while individual nanotubes or their very small aggregates $(<2-3 \mu \mathrm{m}$, invisible with optical microscope) were distributed in the composite matrix. Thus, with ultrasonic processing for up to 10 minutes, a large mass fraction of MWCNTs is concentrated in aggregates, and the content of individual nanotubes and their small aggregates in the composite matrix does not grow significantly. With increasing sonication time in the range of 10-45 minutes, grinding of large aggregates and redistribution of nanotubes in the composite is observed due to their dispersion in the polymer matrix in the form of single nanotubes and small aggregates, which is accompanied by increase in the color intensity of the polymer matrix between the aggregates.
SEM also showed that the size of the aggregates decreased whereas their number and the number of individual nanotubes observed on the composite fracture surfaces increased when $t_{\mathrm{s}}$ grew in the range of 3-10 min. When $t_{\mathrm{s}}$ was increased to $20-45 \mathrm{~min}$ the MWCNT aggregates practically disappeared from the surface of the breaks, and the uniformity of the MWCNT distribution on break surface improved. The observed change of the break structure observed as more frequent location of steps with growing ultrasonic treatment time is another indicative of more uniform MWCNT distribution in the bulk of the PMMA matrix (Figure 2).

\subsection{Conductivity of the composites}

Preliminary studies of the percolation threshold of composites with different MWCNT contents were carried out to provide the maximum sensitivity of the current-voltage characteristics (CVC) of the composites to changes in their structure (Figure 4). The calculation of the conductivity of composites was carried out according to the third measurement of the $\mathrm{CVC}$ at $E=1 \mathrm{~V} / \mathrm{mm}$. It was found that the conductivity of composites increases significantly in the range of MWCNTs concentration 1-2.5 wt\% (0.64$1.6 \mathrm{vol} \%)$. Approximation of the experimental data using the function $\sigma \sim\left(P-P_{\mathrm{c}}\right)^{\mathrm{t}}$ showed that the critical concentration (percolation threshold) $P_{\mathrm{c}}=$ $1.492 \mathrm{wt} \%$ (0.932 vol\%) and $t=1.54$ (Figure 4, Insert). Thus, further studies of the effect of sonication time on the properties of the composites were carried

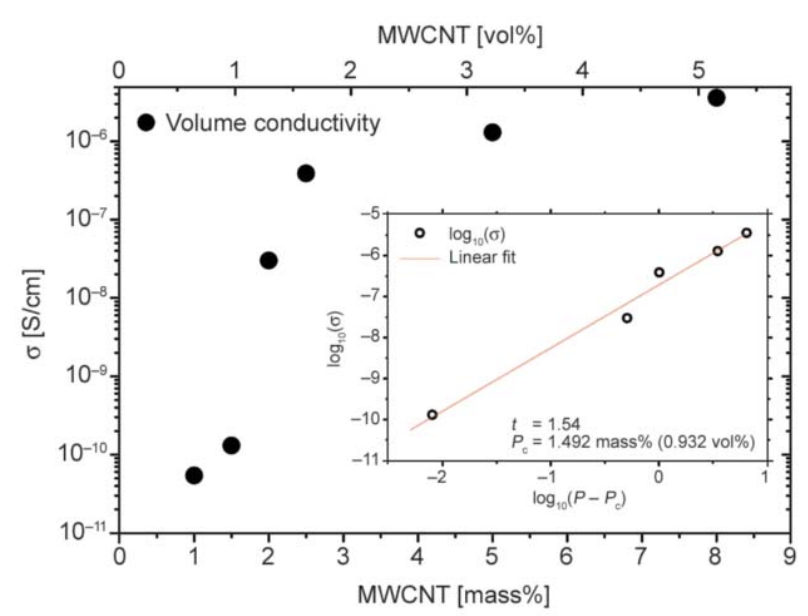

Figure 4. Conductivity of composites versus mass and volume concentration of MWCNTs (data corresponding to the third measurement of CVC at $E=$ $1 \mathrm{~V} / \mathrm{mm})$. Insert shows the fit of the conductivity data using function $\sigma \sim\left(P-P_{\mathrm{c}}\right)^{\mathrm{t}}$ with logarithmic coordinates. 
out at a content of MWCNTs in the composite of $1.5 \mathrm{wt} \%$.

Current-voltage characteristics (CVC) of the composites obtained during successive cyclic measurements of the passing current as a function of applied voltage are shown in Figure 5. The appearance of the initial curve of the current dependence on voltage most significantly differs from the ones obtained in subsequent experiments. At low applied field $(E<$ $3 \mathrm{~V} / \mathrm{mm}$ ), the current determined during the first measurement was lower by $1.5-5$ orders of magnitude than the ones obtained in the subsequent measurements. For each sample it is possible to determine $E_{\text {cr, }}$, where the $I(E)$ dependence deviates from the ohmic one. In the field strength range $0-E_{\text {cr }}$ the dependence $I \sim E^{\mathrm{n}}$, where $n=0.98-1.1$ was observed. Further increase of the applied voltage led to faster current growth than it would be observed if the Ohm law was followed. This result suggested that additional current pathways appear.

A significant current increase with the voltage growth during the first $I(E)$ measurement at $E>10 \mathrm{~V} / \mathrm{mm}$ was found to be typical for all the composites. At $E>100 \mathrm{~V} / \mathrm{mm}$ the power $n$ in $I \sim E^{\mathrm{n}}$ dependence was equal to 2.38, 3.64 and 3.93 for samples $\mathbf{C 2 0}$, C30 and C45, respectively. During subsequent measurements the ohmic conductivity at low voltage substantially increased and the current growth at

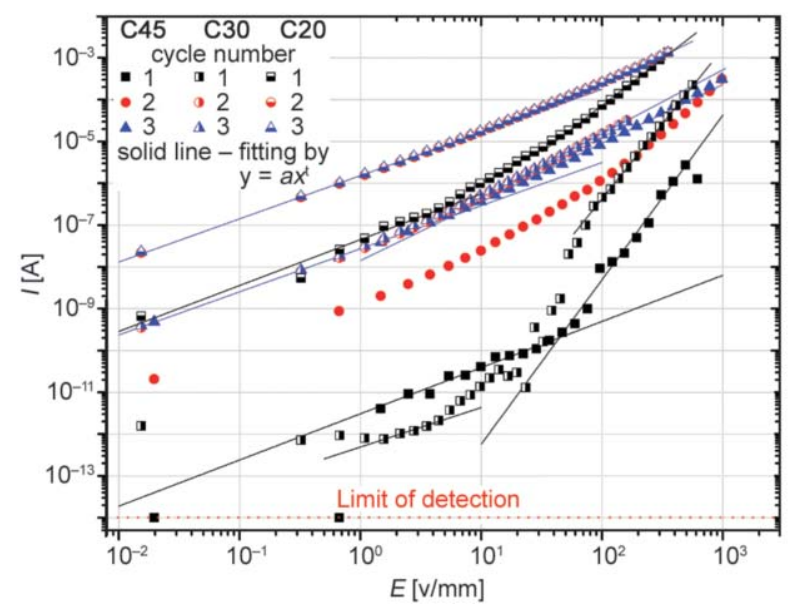

\begin{tabular}{|l|c|c|c|c|}
\hline Sample & Cycle & $\boldsymbol{n}$ LV & $\boldsymbol{n} \mathrm{HV}$ & $\begin{array}{c}\boldsymbol{E}_{\mathrm{cr}} \\
{[\mathbf{V} / \mathbf{m m}]}\end{array}$ \\
\hline \multirow{2}{*}{ C20 } & 1 & 1.10 & 2.34 & 5.48 \\
\cline { 2 - 5 } & 3 & 1.01 & 1.22 & 9.26 \\
\hline \multirow{2}{*}{$\mathbf{C 3 0}$} & 1 & 0.98 & 3.64 & 4.44 \\
\cline { 2 - 5 } & 3 & 1.07 & 1.48 & 5.29 \\
\hline \multirow{2}{*}{$\mathbf{C 4 5}$} & 1 & 1.10 & 3.93 & 43.50 \\
\cline { 2 - 5 } & 3 & 1.03 & 1.40 & 5.81 \\
\hline
\end{tabular}

Figure 5. Curves of three successive $\mathrm{CVC}$ measurements for samples C20, C30 and C45. high voltages decreased. For $\mathbf{C 2 0}$ and $\mathbf{C 3 0}$ the results of the third measurement were the same as those of the second one. The third measurement for $\mathbf{C 4 5}$ gave the same results as the second and third measurements for C30. Meanwhile, $\mathrm{n}$ was in the range of 1.01-1.07 at low voltages and between 1.22 and 1.48 at high voltages. $E_{\mathrm{cr}}$ decreases from 9.26 to $5.29 \mathrm{~V} / \mathrm{mm}$ with an increase of the ultrasonic treatment time. The $I(E)$ dependences obtained during the following measurements practically matched the results of the second and third measurements.

To explain the observed effects, it is necessary to take into account specific features of the composite synthesis by coagulation technique. Contacts between nanotubes are formed during coagulation of MWCNT and the polymer matrix. If MWCNTs are in aggregates or directly contact by their external layers, the contacts between the nanotubes are ballistic. If MWCNTs are predominantly dispersed in PMMA, most likely, the contacts are formed by nanotubes separated by a film of the polymer molecules. Such contacts were characterized as tunnel [32-34]. Their resistivity reaches $10^{10}-10^{11} \Omega$ with potential barrier 2.5-4 eV (typical for polymers). The distance between the nanotubes in such contacts is about 1.0$1.1 \mathrm{~nm}$, which corresponds to 1 polymer molecule in the gap between the nanotubes. In [35] it was found that at random nanotube orientation ballistic contacts give the main contribution to the total conductivity of the composite whereas that of the tunnel ones was about $10 \%$. Ballistic and tunnel contacts are also called ohmic. They determine the composite conductivity at room temperature and low applied voltage. Introduction of 2-3 chains of the polymer molecules between nanotubes increases the contact distance to $1.5-1.8 \mathrm{~nm}$ and resistance to $10^{16} \Omega$. This makes it possible to neglect the contribution of such contacts to ohmic conductivity at low applied voltage or pressure. It was shown [36] that for such contacts the following conductivity mechanisms were possible: space-charge limited current, fluctuation-induced tunneling [37, 38], Coulomb gap [39-41], Coulomb blockade [42-44] and others. For such mechanisms there is a critical value of $E_{\mathrm{cr}}$ correlating with the potential barrier between the contacts. When it is exceeded, non-ohmic dependence of the current on voltage is observed.

So, contacts between the nanotubes formed during the composite synthesis can be divided into two groups: ohmic (ballistic and tunnel) with the distance 
etween the nanotubes $0.34-1.1 \mathrm{~nm}$ and non-ohmic where this distance exceeds $1.1 \mathrm{~nm}$. For a non-ohmic contact, the possibility of participation in the current will be determined by external impact (e.g. by electric field or pressure).

As the surface of MWCNTs used in this study is hydrophobic, during coagulation it is energetically more favorable for the nanotubes to be covered by the hydrophobic PMMA matrix rather than contact with water. Therefore, the main parameter controlling the ratio of MWCNT sections covered with the polymer and nanotubes free from it is the ratio of adhesion energies of PMMA and water to the surface of the nanotubes. For example, the adhesion energy of polystyrene (PS) is higher than that of PMMA. As a result, the percolation threshold (measured at low test voltages) was higher for composites based on PS in comparison with the ones based on PMMA [22]. This is caused by the fact that the resistivity of ohmic contacts between individual nanotubes exceeds that of the nanotubes themselves by a factor of $10^{2}-10^{6}$ [45]. So, the resulting resistivity of the composite is determined by the resistivity of the contacts. For a composite with MWCNT concentration close to the percolation threshold, the total resistivity of the conducting chain $\left(R_{\mathrm{tot}}\right)$ consisting of $n$ nanotubes with the average distance between contacts belonging to one nanotube $\left(L_{\mathrm{m}}\right)$ will be determined by the contact resistances between nanotubes $\left(R_{\mathrm{CN}}\right)$ connected in series $R_{\mathrm{tot}}=(n-1) R_{\mathrm{CN}}$. Meanwhile, the MWCNT aggregate can be described as a large number of parallel resistances $R_{\mathrm{ag}}=R_{\mathrm{CN}} / m$, where $m$ is the number of parallel contacts between nanotubes in the aggregate. So, substitution of two directly contacting nanotubes in the conducting chain of the aggregate will lead to the decrease of its resistance by $R_{\mathrm{CN}}(1-1 / m)$. When the size of the aggregates grows, this effect will increase. However, when the size of the aggregates decreases below $2 L_{\mathrm{m}}$ introduction of aggregates into the conducting chain will not affect its resistance.

The effects observed during the CVC measurements of composites can be explained taking into account these considerations. During the first measurement a substantial increase of the current through the sample was observed for $\mathbf{C 3 0}$ and $\mathbf{C 4 5}$ at large $E$, and this effect was not observed at subsequent measurements. During the following measurement the $I(E)$ dependence was reproducible, and sections typical for ohmic $(n=1-1.2)$ and non-ohmic $(n>1.2)$ conductivity can be distinguished. Therefore, an electrical or thermal breakdown of the polymer layer separating nanotubes occurs during the first measurement as the local strength of the field between MWCNTs on nonohmic contacts can exceed $10^{8} \mathrm{~V} / \mathrm{m}$ due to small distance between the nanotubes. Electrical breakdown of the matrix accompanied by the heating of the contact area and causing local decrease of the polymer viscosity can lead both to squeezing of the polymer chains from the space between the nanotubes due to the action of the static pressure of the polymer matrix from colder sections of the composite and to destruction of the polymer chain structure with the release of gaseous molecules and the formation of conductive carbon fragments. Depending on the distance between the nanotubes in the initial non-ohmic contact, both ohmic (ballistic or tunnel) and non-ohmic contacts with shorter distance between the nanotubes can be formed during the breakdown (due to approaching of the nanotubes under the pressure or due to partial filling of the contact by conductive products of the polymer destruction). The effect of the contact formation is most evident for composite $\mathbf{C 4 5}$. In this case more uniform MWCNT dispersion and coverage of their surface by the polymer chains were observed as a result of prolonged ultrasonic treatment. For example, comparison of the first three CVC for $\mathbf{C 2 0}$ showed less significant changes in its conductivity. This result suggests that fewer non-ohmic contacts capable for transformations a result of a breakdown are present in this sample, i.e. distance between nanotubes in non-ohmic contacts is longer.

The value $n=1,22-1,48$ at $E>E_{\text {cr }}$ indicates that the deviation from the ohmic conductivity mechanism is apparently caused by the presence of a potential barrier in non-ohmic contacts appearing due to ionization of the nanotube-polymer boundaries. The $E_{\mathrm{cr}}$ value correlates with its height and characterizes effective distance between nanotubes in non-ohmic contact.

\subsection{Effect of current on the volume of composites and effect of external pressure on their conductivity}

During electric conductivity measurements of the composites using $E>0.33 \mathrm{~V} / \mathrm{mm}$, they experienced heating and thermal expansion registered using a pressure sensor. Data on changes of the internal pressure in composite $\mathbf{C 2 0}$ (after CVC measurements) during measurement of its resistivity are presented 

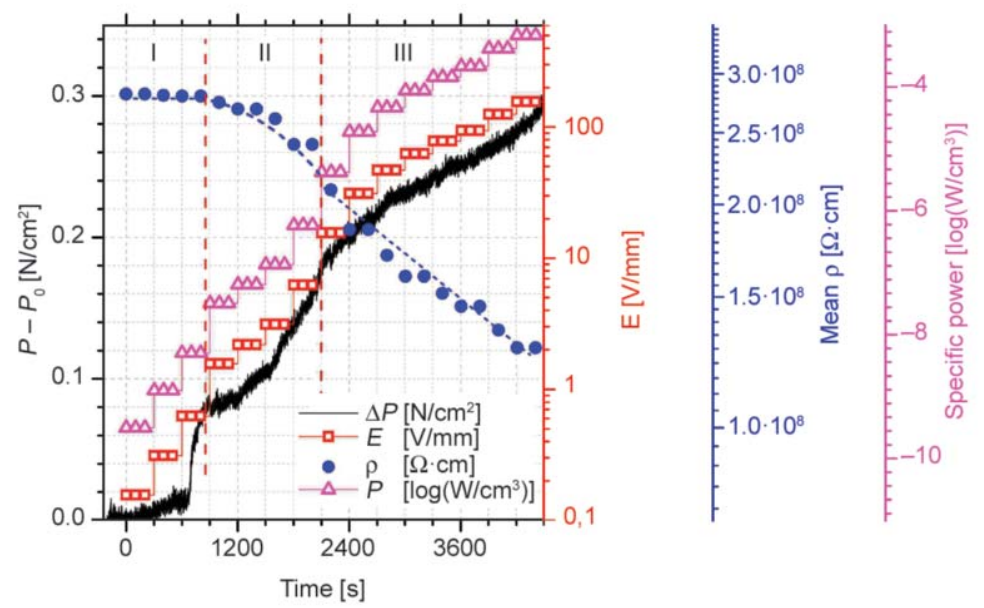

Figure 6. Change of internal pressure in $\mathbf{C 2 0}$ composite (after CVC measurements) depending on the applied voltage during the resistivity measurement $\left(P_{0}=70.7 \mathrm{~N} / \mathrm{cm}^{2}\right)$.

in Figure 6. These results motivated us to estimate change of the composite resistivity at constant applied voltage when the composite was subjected to external pressure. Data illustrating the effect of pressure (in the range of $0-5 \mathrm{MPa}$ ) on the resistivity of the $\mathbf{C 2 0}$ composite are shown in Figure 7. In both case the resistivity of the composite $(\mathbf{C 2 0})$ varied within the same order of magnitude. Taking into account the elastic modulus values for PMMA (3-3.5 GPa) and MWCNTs ( 1000 GPa) [46], the expected change of the material properties will be mostly determined by deformation of the polymer matrix.

An increase of the applied voltage leads to the growth of pressure on measuring electrodes in the composite. Meanwhile, the pressure change $\left(P-P_{0}\right)$ and released power $\log (P)$ vary synchronously. So, the pressure increase is likely caused by the growth of the composite volume due to the energy release. An increase of the pressure on electrodes by $0.01-$ $0.02 \mathrm{~N} / \mathrm{cm}^{2}$ was observed even at $E=0.33 \mathrm{~V} / \mathrm{mm}$ (corresponding to the average current density $4 \cdot 10^{-8} \mathrm{~A} / \mathrm{cm}^{2}$ ). As the released power in this case was equal to $1.3 \cdot 10^{-10} \mathrm{~W} / \mathrm{cm}^{3}$, apparently, there are zones with high local temperature in the composite (ohmic contacts), which can account for the local increase of the composite volume. This suggestion is necessary because the average heating of the composite between the electrodes during the period of time when the $0.33 \mathrm{~V} / \mathrm{mm}$ voltage was applied ( $300 \mathrm{~s}$ ) was $\sim 2 \cdot 10^{-7}{ }^{\circ} \mathrm{C}$, and could not account for the pressure increase. The observed phenomenon is similar to the one described earlier [47] where the observed increase of the composite dimensions was attributed to heat release by a nanotube network. A sharp pressure increase was observed at $E=0.66 \mathrm{~V} / \mathrm{mm}$ indicating that the composite volume grew due to the polymer expansion. At $337 \mathrm{~K}$ excitation of $\beta$-relaxation in PMMA was observed [48]. It could explain the observed pressure increase and give an idea of the local contact overheating magnitude. The $\rho(E)$ dependence has three typical regions. At the first region $\rho$ does not change, i.e. no change of contacts in the composite occurs. At the second region the resistivity goes down. Its decrease correlates with a sharp increase of the pressure in the composite, which can be caused by local overheating and exclusion of the polymer from tunnel contacts between nanotubes and their transformation into ballistic contacts. At the third region $\log (\rho)$ and $\left(P-P_{0}\right)$ linearly decrease with $\log (E)$ growth. The beginning of this region corresponds to $E_{\mathrm{cr}}$ determined from CVC measurements at $9.26 \mathrm{~V} / \mathrm{mm}$ and electrode pressure 1$1.5 \mathrm{~N} / \mathrm{cm}^{2}$ and can be explained by additional current passing through non-ohmic contacts.

The influence of the applied pressure and electric field on the resistance of the $\mathbf{C 2 0}$ composite was studied in the pressure range of $0.5-5 \mathrm{MPa}$ and $E=$ $1-625 \mathrm{~V} / \mathrm{mm}$ (Figure 7). The sample after preliminary CVC measurements was used for this purpose. It was found that for all the dependences $\rho(P)$ obtained, we can distinguish two characteristic regions. In the first section $(0.5-1.5 \mathrm{MPa})$ a significant nonlinear change in resistance from the applied pressure occurs, while in the second section (1.5-5 MPa) a linear dependence of $\log (\rho)$ on the applied pressure is observed (Figure 7a). At the same time, the obtained dependences are well reproduced during repeated successive measurements (3 or more times). The variation of the applied voltage when measuring the dependence of the resistance of the composite on 

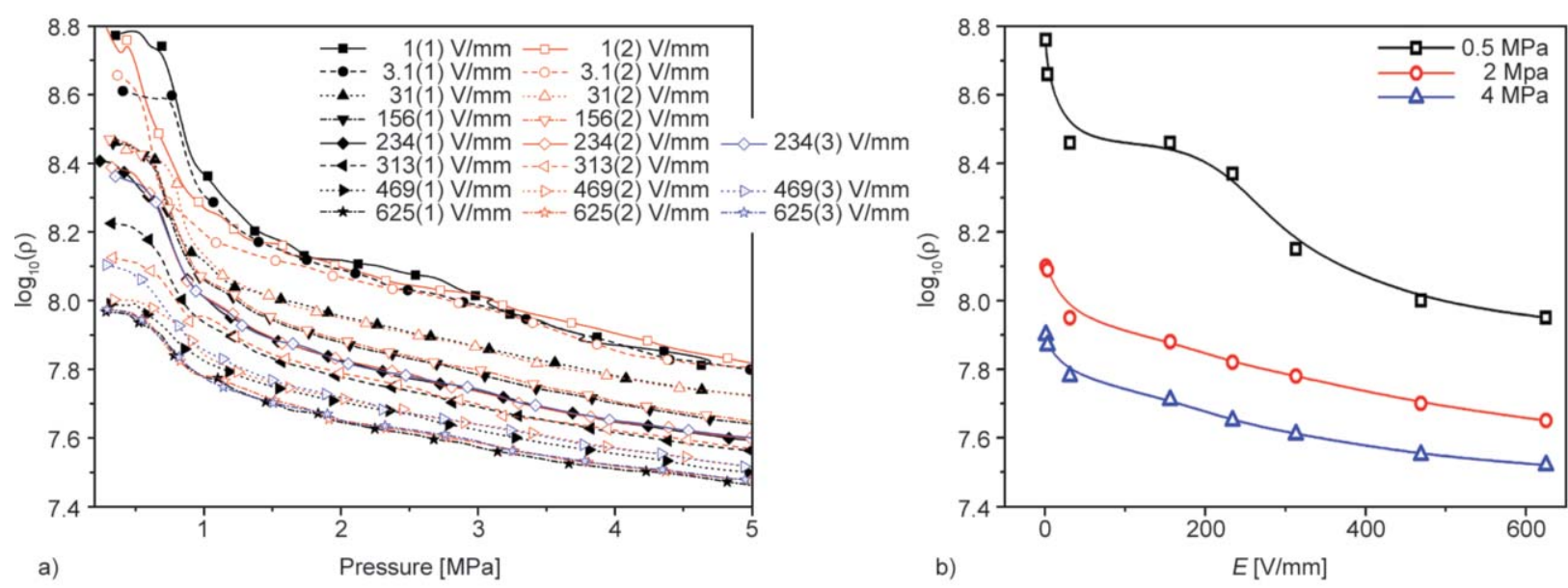

Figure 7. Change of the $\mathbf{C 2 0}$ composite (after CVC measurements) resistivity depending on the applied pressure and voltage. a) The dependence of resistance on pressure at a fixed applied voltage. The number of the sequential test are indicated in parentheses. b) The dependence of resistance on applied voltage at different fixed pressures $(0.5,2$ and $4 \mathrm{MPa})$.

pressure showed that in the range of applied voltages of $1-3.1 \mathrm{~V} / \mathrm{mm}$, the obtained dependences are reproduced in the entire pressure range. A further increase in the applied voltage in the range of $31-625 \mathrm{~V} / \mathrm{mm}$ leads to a decrease in the resistance of the composite in the range of $0.5-1.5 \mathrm{MPa}$ (Figure $7 \mathrm{~b}$ ). Based on the experimental data obtained, it can be concluded that in the $P$ 0-1.5 MPa range, the main contribution to the decrease in resistance is made by the increase in the number of ballistic contacts due to the reversible displacement of polymer from tunnel contacts under the effect of external pressure on the composite. At the same time, the application of a voltage in the range of $1-3.1 \mathrm{~V} / \mathrm{mm}$ leads to a small $\left.\left(\Delta \log _{10}(\rho)\right) \sim 1.5 \%\right)$ decrease in the resistance of the composite in the pressure range of $0.5-1.5 \mathrm{MPa}$ and does not affect the conductivity at a pressure greater than $1.5 \mathrm{MPa}$ (for pressure of $2 \mathrm{MPa} \Delta \log _{10}(\rho)$ ) $0,25 \%$ ) because the applied voltage is lower than a certain earlier for $\mathbf{C 2 0} E_{\mathrm{cr}}=9.26 \mathrm{~V} / \mathrm{mm}$. Increasing the voltage in the range of $31-625 \mathrm{~V} / \mathrm{mm}$ leads to a decrease in resistance over the entire pressure range. A decrease in resistance change in the $P 0.5-1.5 \mathrm{MPa}$ range can be correlated with partial transformation of tunnel contacts due to the displacement of polymer from tunnel contacts between nanotubes under the action of an applied voltage, and a decrease in resistance in the $P 1.5-5 \mathrm{MPa}$ range indicates activation of non-ohmic contacts. Because measurements are reproduced, it can be concluded that the processes of transformation of tunnel contacts into ballistic and activation of nonohmic contacts are reversible. Thus, the following conclusion can be made based on the CVC measurements and obtained $P(E)$ and
$\rho(P, E)$ dependences. When measuring the resistance in a composite, a significant change in its structure occurs, accompanied by the formation of new ballistic (ohmic and tunnel) contacts due to electrical or thermal breakdown of non-ohmic contacts between nanotubes. The number of contacts formed depends on the method of preparation of the composite and the conductivity measurement conditions. This can lead to a decrease in the resistance of the composite up to $10^{5}$ times in the measurement process. During subsequent measurements, the mechanical deformation of the composite occurs, which is reflected in a change in the external dimensions of the sample, which is fixed even at an applied voltage of $0.33 \mathrm{~V} / \mathrm{mm}$ and a current density of $4 \cdot 10^{-8} \mathrm{~A} / \mathrm{cm}^{2}$. Mechanical deformation can lead to the formation of ohmic contacts due to the squeezing of the polymer matrix from the tunnel contacts and a decrease in the measured resistance of the composites.

So, for correct comparison of the effect of the composite structure on specific resistivity, it is more correct to analyze the data obtained during the third CVC measurement. They allow one to estimate the conductivity of the composites with the account of adjacent nanotubes separated by several polymer chains, which are revealed during the first measurement only at high voltages. Variation of the specific resistivity of composites or determination of its precise value could be another practical application of the used method for activation of isolated contacts between MWCNTs. It can be needed for the formation of composites with regulated transmission/reflection of electromagnetic irradiation, for development of sensors with regulated sensitivity, etc. 


\subsection{Conductivity of composites after several CVC measurement cycles}

As it was shown above, during the first 1-3 measurements of CVC, a significant change in the resistivity of the composites may occur due to the formation of new contacts between MWCNTs. After several cycles of measuring the conductivity of MWCNTPMMA composites (CVC measurements), the $I(E)$ and $\rho(E)$ dependences were reproduced quite well. This result indicates that internal stresses in the polymer composites are removed, and they reach a steady state (in the range of currents passing through the composites at applied voltage in the range of 0 $\left.10^{3} \mathrm{~V} / \mathrm{mm}\right)$. The $I(E)$ and $\rho(E)$ dependences in logarithm coordinates obtained in the third CVC measurement for all composites are shown in Figure 8a and 8 b. Two linear sections $\log (I)-\log (E)$ can be distinguished for each composite. Approximation with a function $I \sim E^{\mathrm{n}}$ gives $n=0.98-1.07$ at low $E$ and $1.22-1.52$ at high $E$. Meanwhile $E_{\text {cr }}$ depends nonlinearly on the ultrasonic treatment time (Table 1). As $n \sim 1$ at $E<E_{\text {cr, }}$, in this range the conductivity is determined only by ohmic contacts between nanotubes. At $E>E_{\text {cr }}$ the conductivity of non-ohmic contacts is activated under electric field. The presented $\rho(E)$ dependences demonstrate that $\rho(E)=$ const for all the composites at $E<\sim 1 \mathrm{~V} / \mathrm{mm}$. So, $E=1 \mathrm{~V} / \mathrm{mm}$ was chosen for correct comparison of the sample conductivity.

The obtained dependences of resistivity at $E=$ $1 \mathrm{~V} / \mathrm{mm}$ and $E_{\mathrm{cr}}$ on the sonication time and delivered specific ultrasound energy $\left(J_{\mathrm{s}}\right)$ are presented in Figure $8 \mathrm{c}$. The resistivity of the composites did not
Table 1. Effect of ultrasonic treatment time on the main parameters of the composites determined from the CVC curves.

\begin{tabular}{|l|c|c|c|}
\hline Sample & $\boldsymbol{n} \mathbf{L V}$ & $\boldsymbol{n} \mathbf{H V}$ & $\begin{array}{c}\boldsymbol{E}_{\text {cr }} \\
{[\mathbf{V} / \mathbf{m m}]}\end{array}$ \\
\hline $\mathbf{C 3}$ & 1.02 & 1.22 & 2.52 \\
\hline $\mathbf{C 5}$ & 0.98 & 1.30 & 2.21 \\
\hline $\mathbf{C 7 . 5}$ & 1.01 & 1.26 & 3.17 \\
\hline $\mathbf{C 1 0}$ & 1.02 & 1.52 & 9.14 \\
\hline $\mathbf{C 2 0}$ & 1.01 & 1.22 & 9.26 \\
\hline $\mathbf{C 3 0}$ & 1.07 & 1.48 & 5.29 \\
\hline $\mathbf{C 4 5}$ & 1.03 & 1.40 & 5.81 \\
\hline
\end{tabular}

Here $n \mathrm{LV}$ and $n \mathrm{HV}$ are the exponent indices of $I \sim E^{\mathrm{n}}$ function at low and high $E$ values, respectively.

change much for $t_{\mathrm{s}}=3-20 \mathrm{tmin}$. Meanwhile, a sharp resistivity growth by 1.5 orders of magnitude was observed at $t_{\mathrm{s}}$ in the range of 20-30 min. At $t_{\mathrm{s}}>30 \mathrm{~min}$ the resistivity of the composite practically did not change. The obtained results are in good agreement with the data on the mean length of MWCNTs and composite structure obtained by SEM and optical microscopy and the suggested model explaining the effect of aggregates on the conductivity of the composites.

Thus, for composites obtained at $t_{\mathrm{s}}$ of 3 and $5 \mathrm{~min}$ $\left(J_{\mathrm{s}}=400-800 \mathrm{~J} / \mathrm{cm}^{3}\right)$, a slight increase in ohmic conductivity is observed, which is caused by the destruction of a part of small aggregates $(5-30 \mu \mathrm{m})$ into separate nanotubes with the mean length of $\sim 1.6 \mu \mathrm{m}$ $(0.5-7 \mu \mathrm{m})$, which provides the uniform distribution of MWCNTs contained in them in the polymer matrix, which leads to the formation of conductive paths in the composite volume - the composite begins to
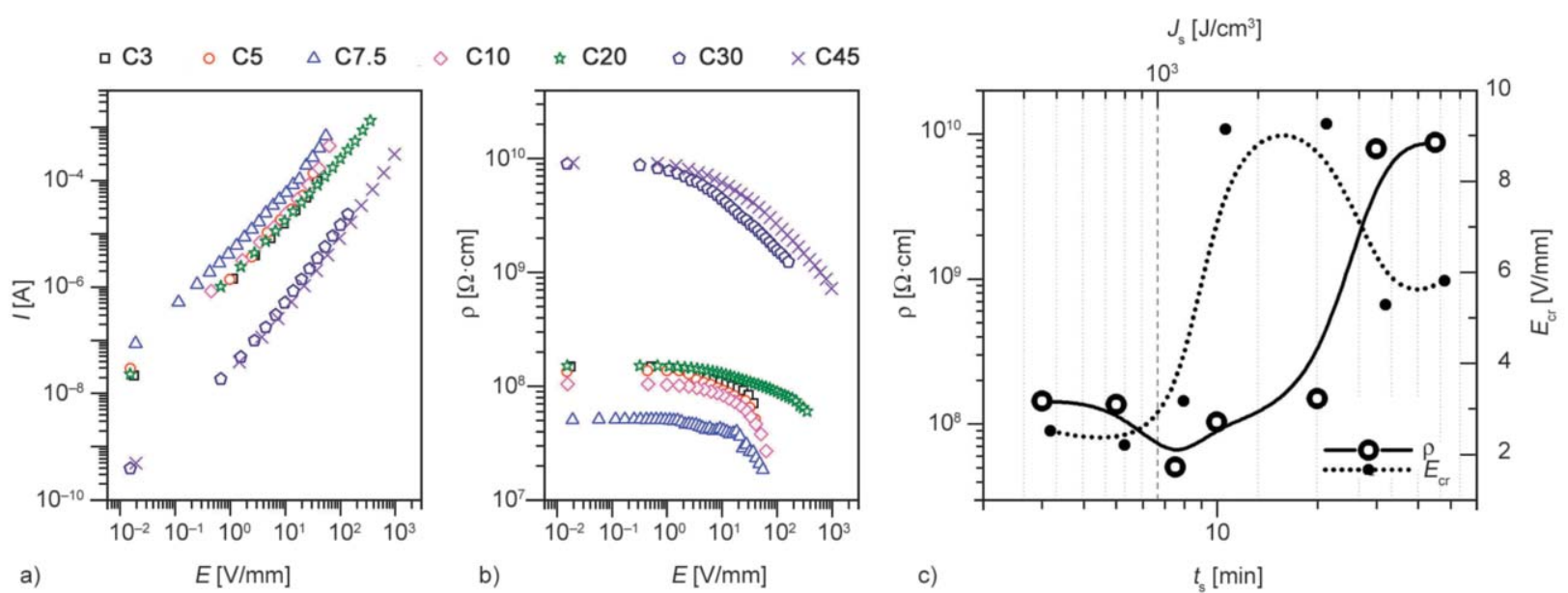

Figure 8. Effect of ultrasonic treatment time on specific resistivity of the composites ( $3^{\text {rd }}$ cycle). (a) CVC of the composites, (b) dependence of specific composite resistivity on the applied voltage, (c) dependence of the ultrasonic treatment time (delivered energy) during synthesis of the composites on specific ohmic resistivity (at $E<\sim 1 \mathrm{~V} / \mathrm{mm}$ ) and $E_{\mathrm{cr}}$ of the composites. 
conduct a noticeable current. The MWCNT aggregates in this case are included into conducting chains. It compensates the low conductivity of the nanotubes in the composite matrix. Ecr does not change much in this range as structural changes of the composite are insignificant.

With an increase in $t_{\mathrm{s}}$ in the range of 7.5-20 min $\left(J_{\mathrm{s}}=1-3 \mathrm{~kJ} / \mathrm{cm}^{3}\right)$, significant changes in the structure of the forming composites occur: a decrease in the share of nanotubes in the aggregates and their redistribution in the polymer matrix is observed. At the same time, the mean length of MWCNTs decreases in the range of $1.63-1.55 \mu \mathrm{m}$, and the mass fraction of the longest nanotubes $(5-7 \mu \mathrm{m})$ decreases by a factor of 2 compared with sample $\mathbf{C} 3$. The lowest resistance was observed for the composite obtained at $t_{\mathrm{s}}$ 7.5. The increase in $t_{\mathrm{s}}$, leads to a decrease in the mean length of MWCNTs about 5\% and dispersion of nanotubes, contributes to the formation of a larger number of contacts containing polymer molecules. Indeed, the observed increase in $E_{\text {cr }}$ indicates an increase in the potential barrier during the formation of conductive paths and an increase in the distance between the nanotubes in non-ohmic contacts.

For the composites produce using $t_{\mathrm{s}}$ equal $30-45 \mathrm{~min}$ $\left(J_{\mathrm{s}}=4.5-7 \mathrm{~kJ} / \mathrm{cm}^{3}\right)$, the remnants of large $(30$ $50 \mu \mathrm{m})$ MWCNT aggregates are further destroyed and the nanotubes are uniformly redistributed in the bulk of the polymer matrix. At the same time, the average length of the MWCNTs decreases to 1.0 $1.2 \mu \mathrm{m}$. This indicates that the decomposition of agglomerates is also accompanied by the destruction of nanotubes with a length of $3 \mu \mathrm{m}$ or longer. As a result, the concentration of dispersed MWCNTs in the matrix increases more than twice in comparison with sample C20, and new contacts between nanotubes are formed. However, most of them are nonohmic, as indicated by an increase in ohmic resistance of composites. As the Ecr decrease was observed for composites C30-C45, the distance between nanotubes in them must be shorter than in composites C10-C20. On the other hand, the decrease of the composite ohmic conductivity indicates that mostly non-ohmic contacts are formed, which is consistent with a decrease in the mean length of MWCNTs. Minor $E_{\mathrm{cr}}$ growth in the $t_{\mathrm{s}}$ range of 30-45 min can be explained by decrease in the mean length of MWCNTs in range 1.5-1.0 $\mu \mathrm{m}$ during the ultrasonic treatment, which is observed by SEM only at sonication times above $20 \mathrm{~min}$. Alternatively, the Ecr increase could be caused by an increase of the polymer layer thickness in non-ohmic contacts as the ohmic conductivity does not significantly change.

\section{Conclusions}

In this study we suggested a method for evaluating the uniformity of the nanotube distribution in the composite based on sequential measurements of the CVC characteristics of composites in a wide range of applied voltages (up to $10^{3} \mathrm{~V} / \mathrm{mm}$ ). In a composite with uniform distribution of nanotubes, large number of non-ohmic contacts leads to substantial increase of the specific resistivity to $10^{13}-10^{14} \Omega \cdot \mathrm{cm}$. This makes it difficult to measure it at low $E$ and impairs reproducibility of the results because partial transformation of contacts due to the heat release under electrical current takes place during the measurements already at $E=0.3 \mathrm{~V} / \mathrm{mm}$ and current density $4 \cdot 10^{-8} \mathrm{~A} / \mathrm{cm}^{2}$, while in the case of a high applied voltage, the decrease in resistance can reach $10^{5}$ due to the formation of new contacts between nanotubes. It was suggested to perform controlled activation of non-ohmic contacts between adjacent nanotubes in electric field below $1 \mathrm{kV} / \mathrm{mm}$ and control reproducibility of the resistivity measurements using CVC. Using this method, it was found that the critical voltage characterizes the change in mechanism of conductivity, agrees well with the uniform of distribution of MWCNT in the composite, provided that the mean length of the nanotubes is constant. Ultrasonic treatment of MWCNT suspension for at least $20 \mathrm{~min}$ (delivered energy $J_{\mathrm{s}} \sim 3 \mathrm{~kJ} / \mathrm{cm}^{3}$ ) was required to achieve uniform distribution of nanotubes in MWCNT-PMMA composites obtained by coagulation technique. The dependence of the $\rho(P)$ varies from the applied voltage. In the case of $E<E_{\text {cr }} \rho(P)$ does not depend on the applied voltage. It is assumed that in the case of pressure acting on the composite at constant voltage, the change in conductivity is mainly caused by the reversible displacement of polymer molecules from the contact area. In the case of $E>E_{\text {cr }} \rho(P)$ an increase in the applied voltage leads to the activation of tunnel contacts with simultaneous activation of non-contact contacts and an increase in the internal pressure in the composite. The suggested method for activation of isolated contacts between nanotubes can be used for modification of MWCNT based composites or obtaining required 
specific resistivity in them for production of functional materials or devices on their basis (e.g. pressure sensors or deformation sensors, etc.).

\section{Acknowledgements}

This work was supported by the Russian Science Foundation (grant 17-73-20293),

\section{Reference}

[1] Dresselhaus M. S., Dresselhaus G., Charlier J. C., Hernández E.: Electronic, thermal and mechanical properties of carbon nanotubes. Philosophical Transactions of the Royal Society A: Mathematical, Physical and Engineering Sciences, 362, 2065-2098 (2004). https://doi.org/10.1098/rsta.2004.1430

[2] Popov V. N.: Carbon nanotubes: Properties and application. Materials Science and Engineering R: Reports, 43, 61-102 (2004). https://doi.org/10.1016/j.mser.2003.10.001

[3] Feller J-F., Castro M., Kumar B.: Polymer-carbon nanotube conductive nanocomposites for sensing. in 'Polymer-carbon nanotube composites' (eds.: McNally T., Pötschke P.) Woodhead, Cambridge, 760-803 (2011). https://doi.org/10.1533/9780857091390.3.760

[4] Mittal V.: Polymer nanotube nanocomposites: Synthesis, properties, and applications. Wiley, New York (2014).

[5] Georgousis G., Pandis C., Kalamiotis A., Georgiopoulos P., Kyritsis A., Kontou E., Pissis P., Micusik M., Czanikova K., Kulicek J., Omastova M.: Strain sensing in polymer/carbon nanotube composites by electrical resistance measurement. Composites Part B: Engineering, 68, 162-169 (2015).

https://doi.org/10.1016/j.compositesb.2014.08.027

[6] Alamusi, Hu N., Fukunaga H., Atobe S., Liu Y., Li J.: Piezoresistive strain sensors made from carbon nanotubes based polymer nanocomposites. Sensors, 11, 10691-10723 (2011). https://doi.org/10.3390/s111110691

[7] Obitayo W., Liu T.: A review: Carbon nanotube-based piezoresistive strain sensors. Journal of Sensors, 2012, 652438/1-652438/15 (2012).

https://doi.org/10.1155/2012/652438

[8] Al-Saleh M. H., Saadeh W. H., Sundararaj U.: EMI shielding effectiveness of carbon based nanostructured polymeric materials: A comparative study. Carbon, 60, 146-156 (2013). https://doi.org/10.1016/j.carbon.2013.04.008

[9] Chung D. D. L.: Electromagnetic interference shielding effectiveness of carbon materials. Carbon, 39, 279-285 (2001). https://doi.org/10.1016/S0008-6223(00)00184-6

[10] González M., Pozuelo J., Baselga J.: Electromagnetic shielding materials in $\mathrm{GHz}$ range. The Chemical Record, 18, 1000-1009 (2018). https://doi.org/10.1002/tcr.201700066
[11] Zaporotskova I. V., Boroznina N. P., Parkhomenko Y. N., Kozhitov L. V.: Carbon nanotubes: Sensor properties. A review. Modern Electronic Materials, 2, 95-105 (2016). https://doi.org/10.1016/j.moem.2017.02.002

[12] Bauhofer W., Kovacs J. Z.: A review and analysis of electrical percolation in carbon nanotube polymer composites. Composites Science and Technology, 69, 14861498 (2009). https://doi.org/10.1016/j.compscitech.2008.06.018

[13] Spitalsky Z., Tasis D., Papagelis K., Galiotis C.: Carbon nanotube-polymer composites: Chemistry, processing, mechanical and electrical properties. Progress in Polymer Science, 35, 357-401 (2010).

https://doi.org/10.1016/j.progpolymsci.2009.09.003

[14] Sarychev A. K., Shalaev V. M.: Electrodynamics of metamaterials. World Scientific Publishing, Singapore (2007).

[15] Aguilar J. O., Bautista-Quijano J. R., Avilés F.: Influence of carbon nanotube clustering on the electrical conductivity of polymer composite films. Express Polymer Letters, 4, 292-299 (2010).

https://doi.org/10.3144/expresspolymlett.2010.37

[16] Saphiannikova M., Skipa T., Lellinger D., Alig I., Heinrich G.: Superposition approach for description of electrical conductivity in sheared MWNT/polycarbonate melts. Express Polymer Letters, 6, 438-453 (2012). https://doi.org/10.3144/expresspolymlett.2012.47

[17] Aravand M., Lomov S. V., Verpoest I., Gorbatikh L.: Evolution of carbon nanotube dispersion in preparation of epoxy-based composites: From a masterbatch to a nanocomposite. Express Polymer Letters, 8, 596-608 (2014). https://doi.org/10.3144/expresspolymlett.2014.63

[18] Chen S. J., Zou B., Collins F., Zhao X. L., Majumber M., Duan W. H.: Predicting the influence of ultrasonication energy on the reinforcing efficiency of carbon nanotubes. Carbon, 77, 1-10 (2014).

https://doi.org/10.1016/j.carbon.2014.04.023

[19] Peponi L., Puglia D., Torre L., Valentini L., Kenny J. M.: Processing of nanostructured polymers and advanced polymeric based nanocomposites. Materials Science and Engineering R: Reports, 85, 1-46 (2014).

https://doi.org/10.1016/j.mser.2014.08.002

[20] Fromyr T-R., Bourgeaux-Goget M., Hansen F. K.: A comparative study of the dispersion of multi-wall carbon nanotubes made by arc-discharge and chemical vapour deposition. Journal of Nanoscience and Nanotechnology, 15, 3496-3506 (2015).

https://doi.org/10.1166/jnn.2015.9860

[21] Chang C-M., Liu Y-L.: Electrical conductivity enhancement of polymer/multiwalled carbon nanotube (MWCNT) composites by thermally-induced defunctionalization of MWCNTs. ACS Applied Materials Interfaces, 3, 2204-2208 (2011). https://doi.org/10.1021/am200558f 
[22] Mazov I., Kuznetsov V., Romanenko A., Suslyaev V.: Properties of MWNT-containing polymer composite materials depending on their structure. in 'Composites and their properties' (ed.: Hu N.) InTech, Rijeka, 3760 (2012).

https://doi.org/10.5772/48245

[23] Hu C. H., Liu C. H., Chen L. Z., Fan S. S.: Semiconductor behaviors of low loading multiwall carbon nanotube/poly(dimethylsiloxane) composites. Applied Physics Letters, 95, 103103/1-103103/3 (2009).

https://doi.org/10.1063/1.3223777

[24] Liu C. H., Fan S. S.: Nonlinear electrical conducting behavior of carbon nanotube networks in silicone elastomer. Applied Physics Letters, 90, 041905/1-041905/3 (2007). https://doi.org/10.1063/1.2432283

[25] Berliocchi M., Orlanducci S., Reale A., Regoliosi P., Di Carlo A., Lugli P., Terranova M. L., Brunetti F., Bruni G., Cirillo M.: Single wall carbon nanotube based aggregates and their electrical characterization. Synthetic Metals, 145, 171-176 (2004).

https://doi.org/10.1016/j.synthmet.2004.05.012

[26] Bryning M. B., Islam M. F., Kikkawa J. M., Yodh A. G.: Very low conductivity threshold in bulk isotropic single-walled carbon nanotube-epoxy composites. Advanced Materials, 17, 1186-1191 (2005). https://doi.org/10.1002/adma.200401649

[27] Huang Y. Y., Terentjev E. M.: Tailoring the electrical properties of carbon nanotube-polymer composites. Advanced Functional Materials, 20, 4062-4068 (2010). https://doi.org/10.1002/adfm.201000861

[28] Usoltseva A., Kuznetsov V., Rudina N., Moroz E., Haluska M., Roth S.: Influence of catalysts' activation on their activity and selectivity in carbon nanotubes synthesis. Physica Status Solidi (b), 244, 3920-3924 (2007).

https://doi.org/10.1002/pssb.200776143

[29] Kuznetsov V. L., Krasnikov D. V., Schmakov A. N., Elumeeva K. V.: In situ and ex situ time resolved study of multi-component $\mathrm{Fe}-\mathrm{Co}$ oxide catalyst activation during MWNT synthesis. Physica Status Solidi (b), 249, 2390-2394 (2012).

https://doi.org/10.1002/pssb.201200120

[30] Kuznetsov V. L., Bokova-Sirosh S. N., Moseenkov S. I., Ishchenko A. V., Krasnikov D. V., Kazakova M. A., Romanenko A. I., Tkachev E. N., Obraztsova E. D.: Raman spectra for characterization of defective CVD multiwalled carbon nanotubes. Physica Status Solidi (b), 251, 2444-2450 (2014).

https://doi.org/10.1002/pssb.201451195

[31] Du F., Fischer J. E., Winey K. I.: Coagulation method for preparing single-walled carbon nanotube/poly(methyl methacrylate) composites and their modulus, electrical conductivity, and thermal stability. Journal of Polymer Science Part B: Polymer Physics, 41, 3333-3338 (2003). https://doi.org/10.1002/polb.10701
[32] Bao W. S., Meguid S. A., Zhu Z. H., Weng G. J.: Tunneling resistance and its effect on the electrical conductivity of carbon nanotube nanocomposites. Journal of Applied Physics, 111, 093726/1-093726/7 (2012). https://doi.org/10.1063/1.4716010

[33] De Vivo B., Lamberti P., Spinelli G., Tucci V., Vertuccio L., Vittoria V.: Simulation and experimental characterization of polymer/carbon nanotubes composites for strain sensor applications. Journal of Applied Physics, 116, 054307/1-054307/14 (2014). https://doi.org/10.1063/1.4892098

[34] Li C., Thostenson E. T., Chou T-W.: Dominant role of tunneling resistance in the electrical conductivity of carbon nanotube-based composites. Applied Physics Letters, 91, 223114/1-223114/3 (2007).

https://doi.org/10.1063/1.2819690

[35] Gong S., Zhu Z. H., Meguid S. A.: Anisotropic electrical conductivity of polymer composites with aligned carbon nanotubes. Polymer, 56, 498-506 (2015).

https://doi.org/10.1016/j.polymer.2014.11.038

[36] Long Y-Z., Chen Z., Gu C., Wan M., Duvail J-L., Liu Z., Ringer S. P.: A review on electronic transport properties of individual conducting polymer nanotubes and nanowires. in 'Nanowires science and technology' (ed.: Lupu N.) InTech, Rijeka, 223-242 (2010).

https://doi.org/10.5772/39488

[37] Kaiser A. B., Rogers S. A., Park Y. W.: Charge transport in conducting polymers: Polyacetylene nanofibres. Molecular Crystals and Liquid Crystals, 415, 115-124 (2004).

https://doi.org/10.1080/15421400490481421

[38] Kaiser A. B., Park Y. W.: Current-voltage characteristics of conducting polymers and carbon nanotubes. Synthetic Metals, 152, 181-184 (2005).

https://doi.org/10.1016/j.synthmet.2005.07.245

[39] Kang N., Hu J. S., Kong W. J., Lu L., Zhang D. L., Pan Z. W., Xie S. S.: Consistent picture of strong electron correlation from magnetoresistance and tunneling conductance measurements in multiwall carbon nanotubes. Physical Review B, 66, 241403/1-241403/4 (2002). https://doi.org/10.1103/PhysRevB.66.241403

[40] Ma Y-J., Zhou F., Lu L., Zhang Z.: Low-temperature transport properties of individual $\mathrm{SnO} 2$ nanowires. Solid State Communications, 130, 313-316 (2004). https://doi.org/10.1016/j.ssc.2004.02.013

[41] Long Y., Zhang L., Chen Z., Huang K., Yang Y., Xiao H., Wan M., Jin A., Gu C.: Electronic transport in single polyaniline and polypyrrole microtubes. Physical Review B, 71, 165412/1-165412/7 (2005). https://doi.org/10.1103/PhysRevB.71.165412

[42] Saha S. K.: Room-temperature single-electron tunneling in conducting polypyrrole nanotube. Applied Physics Letters, 81, 3645-3647 (2002). https://doi.org/10.1063/1.1519104 
[43] Aleshin A. N., Lee H. J., Jhang S. H., Kim H. S., Akagi K., Park Y. W.: Coulomb-blockade transport in quasione-dimensional polymer nanofibers. Physical Review B, 72, 153202/1-153202/4 (2005). https://doi.org/10.1103/PhysRevB.72.153202

[44] Long Y-Z., Wang W-L., Bai F-L., Chen Z-J., Jin A-Z., Gu C-Z.: Current-voltage characteristics of an individual helical CdS nanowire rope. Chinese Physics B, 17, 1389-1393 (2008).

https://doi.org/10.1088/1674-1056/17/4/039

[45] An L., Friedrich C. R.: Measurement of contact resistance of multiwall carbon nanotubes by electrical contact using a focused ion beam. Nuclear Instruments and Methods in Physics Research Section B: Beam Interactions with Materials and Atoms, 272, 169-172 (2012).

https://doi.org/10.1016/j.nimb.2011.01.058
[46] Elumeeva K. V., Kuznetsov V. L., Ischenko A. V., Smajda R., Spina M., Forró L., Magrez A.: Reinforcement of CVD grown multi-walled carbon nanotubes by high temperature annealing. AIP Advances, 3, 112101/1112101/8 (2013).

https://doi.org/10.1063/1.4829272

[47] Chen L. Z., Liu C. H., Hu C. H., Fan S. S.: Electrothermal actuation based on carbon nanotube network in silicone elastomer. Applied Physics Letters, 92, 263104/1263104/3 (2008). https://doi.org/10.1063/1.2955513

[48] Kranauskaite I., Macutkevic J., Banys J., Kuznetsov V. L., Moseenkov S. I., Rudyna N. A., Krasnikov D. V.: Length-dependent broadband electric properties of PMMA composites filled with carbon nanotubes. Physica Status Solidi (a), 213, 1025-1033 (2016). https://doi.org/10.1002/pssa.201532289 